\title{
Die Bedeutung der Struktur für die Besiedlung von Meeresbiotopen
}

\author{
Adolf Remane \\ Zoologisches Institut der Universität Kiel
}

\begin{abstract}
The significance of the structure for the colonization of marine habitats. The quality and quantity of marine bottom faunas depends to a large extent on the physical structure and chemical properties of the substratum. On rocky bottoms, the surface structure (rough or smooth), its durableness as well as its chemical properties are of special importance. Rocky surfaces may be significantly modified through the action of various organisms. The importance of the structure of the substratum becomes particularly evident in the sandy regions. Within the interstitial system (mesopsammon) between the sand grains lives a rich, special fauna. Its existence and abundance depend largely upon the size and shape of the interstitial spaces, and these in turn, are a function of grain size and grain arrangement. Another important aspect is the stratification. Layers of clay or mud, for example, may make the colonization of an otherwise suitable interstitial habitat impossible. A flocky, fluffy layer of organic detritus, on the other hand, may have positive effects and thus facilitate colonization. Careful consideration of the ecological significance of bottom structures has proven very useful in solving zoogeographic as well as taxonomic problems.
\end{abstract}

Im Gegensatz zu dem Landraum ist der Bezirk des Wassers und speziell des Meeres dreischichtig aufgebaut. Während der Luftbezirk über dem Land kein autonomer Lebensraum ist, da er seine Lebewesen letzten Endes vom Boden des Landes erhält, ist der Wasserbereich des Meeres durch seinen Gehalt an Grundnährstoffen und Produzenten ein relativ autonomer Bezirk, der weitgehend losgelöst von den Komplikationen der 2. und 3. Schicht, nämlich den Grenzbereichen zwischen Wasser und Boden und dem Boden, selbst erforscht werden kann. Das ist wohl der Grund, warum zunächst die Meeresforschung sich diesem Lebensraum zuwandte. Hier schien die Möglichkeit gegeben, fast wie im Experiment in einer Kultur, den Stoffkreislauf von den chemisch-physikalischen Gegebenheiten, ihree Verwertung durch die Produzenten, dieser durch die Konsumenten und zurück durch die Tätigkeit der Reduzenten zur chemischphysikalischen Ausgangssituation entsprechend den Gedanken LIEBIGs vom Stoffkreislauf (vgl. BRANDT 1905) zu untersuchen.

Es war naheliegend, diesen Stoffkreislauf im Meer nicht nur qualitativ, sondern auch quantitativ zu erfassen. So sehr die Biologie der quantitativen, also exakten Fas.sung ihrer Prozesse zustreben muß, so bedenklich ist es, bei diesem Schritt alle die Einzelprozesse zu ubberspringen, die in Einzelarten mit ihrer speziellen Physiologie und Aktivität vor sich gehen, und die in Wirklichkeit die chemischen Fabriken des Stoffkreislaufes sind. Quantitäten von Grundstoffen und Angaben der Biomasse ergeben nur 
ganz grobe Einzeldaten aus dem komplizierten. Wirkungsgefüge des Meeres. Erschwerend kommt hinzu, daß das Pelagial des Meeres nur zum Teil ein unablängiges System ist, in dem die Vorgänge unbeeinflußt von anderen Lebensräumen ablaufen. Gerade im Meer sind die biologischen. Wechselwirkungen zwischen Pelagial und Meeresboden besonders stark. Der überwiegende Teil der Makrofauna des Bodens entsendet pelagische Larven oder eine pelagische Generation ins freie Wasser, die die Biomasse entscheidend beeinflussen können. Andere Gruppen (Peracarida, Polychaeten) dringen in einer Schwärmphase in Massen ins freie Wasser. Ferner kann durch Symbionten wie Zooxanthellen und Zoochlorellen ein interner Stoffkreislauf innerhalb der Organismen auftreten, so daß der Organismus und damit die Biomasse von einer Reihe von Nährstoffen im umgebenden Wasser unabhängig wird (vgl. die Korallenriffe). Solche Symbionten sind im Meere sehr verbreitet (Radiolarien, Cnidarier), sie können eine weite Diskrepanz zwischen den chemischen Faktoren des Wassers und der Biomasse ergeben.

Natürlich sind die Wechselwirkungen im Meeresboden noch viel komplizierter als im Pelagial. Es kommt hier zu den chemischen Faktoren noch ein weiterer hinzu: die Struktur. Sie kann die Qualität und Quantität der Bodenfauna entscheidend beeinflussen. Die Agrarwissenschaft hat seit langem erkannt, daß nicht nur der Chemismus des Bodens Gedeihen und Ertrag bestimmt, sondern auch die Struktur des Bodens. So gibt denn fast jeder Meeresbiologe, wenn er den Lebensraum einer Art charakterisiert, den Untergrund an, eben aus der Erkenntnis heraus, daß die Art des Substrates über Vorkommen und Nichtvorkommen vieler Arten entscheidet. Umgekehrt aber verändern viele Organismen durch ihre Tätigkeit die Struktur des Lebensraumes, so daß dieser ein Wechselsystem zwischen dem Substrat und den Organismen wird.

Ich möchte hier kurz die Richtungen angeben, in denen sich diese Wechselwirkung vollzieht. Der Felsboden ist im Meer Siedlungsraum einer so reichen Fauna und Flora als Epilithion, daß er meist einen hohen Oberzug sessiler Organismen erhält, der aus Balaniden, Polychaeten, Ascidien, Krustenalgen oder Großtangen wie Laminaria, Macrocystis, Durvillea besteht. Da das Substrat hier nur Haftsubstrat ist, scheint seine spezielle Qualität - ob Granit, Buntsandstein, Kalk - für diese Epiflora und Epifauna von geringerer Bedeutung zu sein. Immerhin bestehen Differenzen in der Besiedlung des Substrates. Den Har Tog (1959) hat die günstige Situation an den Deichen Hollands benutzt, um die Verschiedenheiten der Algenassoziation auf verschiedenen Substraten (Granit, Basalt) zu untersuchen. Sie sind gering, und viele Arten wachsen ebenso gut auf Felsboden verschiedener Qualitär, als auch auf Holz oder sogar auf Lehmboden. Fucus wächst beispielsweise in der Bucht von Arcachon auf den Iles des Oiseaux zwischen Andel (Puccinellia), so daß ein Tang der felsigen Meeresküste bis in eine Graswiese vordringt. Ebenso können als Siedler auf Muschelschalen, Algen und anderen Festkörpern epilithische Pflanzen und Tiere bis weit auf Weich- und Sandböden vordringen. Laminaria kann mit Muschelschalen von Arctica und Astarte als Haftsubstrat auskommen, Fucus mit Mytilus, und Spirorbis, Halichondria, Styeolopsis, Ciona sogar mit der Alge Furcellaria.

Soweit es zur Zeit zu beurteilen ist, können 3 Faktoren die Substratbesiedlung beeinflussen: 1. Die Oberfläche des Felsens beziehungsweise des Felskörpers: a) ob rauh oder glatt. Rauhigkeiten an der Oberfläche können die erste Anheftung schwimmender Jugendstadien erleichtern, teilweise erst ermöglichen. Bekannt ist dies vor allem bei 
Balaniden. b) Ob einheitlich oder von Spalten durchsetzt. Solche Spalten sind im Gezeitengebiet lebensfördernd für amphibische oder semiterrestrische Arten, die sich bei extremer Insolation oder Trockenheit (Littorina saxatilis, Ligia, Collembolen, Scolioplanes und andere) in die Spalten zurückziehen. Für diese Arten sind die Spalten ein Fluchtraum. Im Meeresgebiet selbst sind Spalten im Gestein Siedlungsstellen vieler Arten des Weichbodens (sedentäre Polychaeten wie Sabellidae, Spionidae, ferner Pennatularien und andere). 2. Die Dauerhaftigkeit der Oberfläche des Substrates. Die „Verwitterung“ der einzelnen Festböden ist einerseits von ihrer Struktur abhängig (Tuffböden sind rascher vergänglich als Basalt- oder Granitböden), andererseits von der angreifenden Kraft der Wellen. Gerade diesen Faktor können die Besiedler hemmen oder verstärken. Große, von der Brandung bewegte Tangbüsche können die Zugwirkung an der Haftfläche verstärken und so die Abtragung des Gesteins erleichtern und die Existenz der Art an Stellen, an denen diese leicht möglich wäre, gefährden. Hier greifen die physikalischen Faktoren des Milieus ein, die auf gleichartigen Böden eine verschiedene Besiedlung je nach der Kraft des Wellenangriffes bedingen können. 3. Die chemische Beschaffenheit des Substrates. Sicher ist, daß viele Larven oder Jugendstadien sessiler Tiere ein Chemorezeptionsvermögen haben; inwieweit dies für die Wahl des Gesteins als Lebensraum wirksam ist, können erst intensivere Experimente zeigen. Die Verschiedenheit des Substrates, das von vielen sessilen Tieren besiedelt wird (Felsboden, Holz, Algen, Seegras, Muschelschalen), zeigt für viele eine weitgehende Toleranz, andererseits fordert die Spezifiät vieler Epizoen und Epiphyten, besonders der Peritrichen (PRecht et al. 1935), spezielle Reaktionen.

Waren die Strukturbesonderheiten nur in geringfügigem Maße für die Ansiedlung des Felsaufwuchses von Bedeutung, so ist es die Struktur des Aufwuchses, also besonders der Algen in stärkerem Maße. Ich erwähnte schon, daß die Zahl der sessilen Ansiedler auf Algen und Muscheln rasch mit der Größe der Haftmöglichkeiten abnimmt und Algen vom Typ Furcellaria mit 0,5 bis $1 \mathrm{~mm}$ dicken Thalluszweigen die Grenzen für die Existenz einer reicheren Mikrofauna darstellen. Auf feine Algen, zum Beispiel vom Typ Ceramium, gehen noch sessile Arten mit stolonialem Wuchs (Hydroiden, Bryozoen), Schwämme (Halichondria) und natürlich sessile Arten der Mikrofauna (Peritrichen, Suctorien) über. Umgekehrt verhält sich aber die vagile Mikrofauna: Harpacticoidea, Ostracoden, Nematoden, kleine Polychaeten, Halacariden. Ihre Quantität nimmt an fädigen Buschalgen stark zu. Auf Fucus ist die Zahl dieser Arten zwischen den epiphytischen Algen des Tangs (Elachista, Ectocarpus) sehr hoch, aber zwischen den reinen Fucusthalli gering. Als Beispiel führe ich eine Auszählung an. In einer Fucus-Siedlung der Kieler Bucht (Weißenhaus) lebten auf $1 \mathrm{~m}$ Thallus ohne Kleinalgenbewuchs 29 Asseln (Idotea baltica), 32 Amphipoden (Calliopius), eine Anzahl kleiner Mytilus (unter $1 \mathrm{~cm}$ ), 9 Ostracoden (3 Cytherura nigrescens, 3 Cythere, 1 Xestoleberis, 2 Loxoconcha) und etwa 15 Halacariden. An einem Fucusbezirk von $50 \mathrm{~cm}$ Länge, der dicht mit Büschen von Elachista bewachsen war, lebten 95 Idotea (viele kleine Tiere), 5 Jaera, 655 Calliopsis (viele kleine Tiere), 7 Gammarus, 75 Oligochaeten (Paranais), 3 Fabricia, 135 Mückenlarven (Trichocladius), 21 Ostracoden, davon etwa die Hälfte Cythere, 438 Harpacticiden und viele Halacariden.

Sehr deutlich wird die Beziehung Substrat - Besiedlung bei den endolithischen Organismen, die im Gestein bohren. Bei den mechanisch bohrenden Formen (Poly- 
chaeten wie Polydora ciliata, Bohrschwämme wie Clione, Pholadae als Bohrmuscheln) spielt selbstverständlich die „Angreifbarkeit“ des Substrates eine wichtige Rolle. Daher ist der weiche Kalkstein bevorzugt, einschließlich Molluskenschalen; viele Arten siedeln auch auf harten Tonböden und untermeerischem Torf. Auf Helgoland fand ich Polydora, die hier die Kreidefelsen dicht mit Bohrlöchern durchsetzt, im Sandstein nur dort, wo eine Spalte das Eindringen gestattet. Die noch engere Beschränkung der chemisch bohrenden Formen (Lithodomidae, Phoronis) auf Kalkgestein bedarf keiner Begründung. Wohnhöhlen im harten Gestein bauen die „höhlenbewohnenden" Seeigel (Paracentrotus). Thre harten Zähne, die mit einer schmelzartigen Hartschicht versehen sind, ermöglichen eine Nagetätigkeit auch an härteren Gesteinen, sie nagen sogar Eisenteile an. Das Substrat ist bei den holzbohrenden Arten wieder wichtig, hier kann eine Bevorzugung bestimmter Holzarten vorhanden sein, wobei wieder der Härtegrad wichtig ist (vgl. ScHütz 1961). Eine Spezialisierung auf bestimmte Holzarten wie bei den Holzbewohnern des Landes (Insekten) ist aber im Meer keineswegs vorhanden.

Noch wenig untersucht ist aber die biologische Auswirkung der Veränderungen, die die Organismen an ihrem Wohnsubstrat bewirken. Es ist selbstverständlich, daß die epilithischen Tiere und Pflanzen das Verhalten der Wasserschicht oberhalb des Hartbodens beeinflussen, die Pflanzen beispielsweise durch Verringerung der auftretenden Lichtmenge und des Wellenanpralls. Krustenbildende Organismen wie Lithothamnium, Hildenbrandtia, Membranipora und Didemnum können sogar eine völlige Schutzschicht über dem Gestein bilden, deren Wirkung im einzelnen noch zu untersuchen wäre. Der tierische Aufwuchs, der gleichfalls eine geschlossene Schicht auf der Unterlage bilden kann, besteht überwiegend aus Strudlern (Mytilus, Modiolus, Aszidien, Polychaeten, zum Beispiel Fabricia und Serpuliden, und Poriferen) oder Filtrierern (Balaniden), die eine starke Wasserbewegung oberhalb des Substrates bewirken. In ruhigen Meeresbuchten soll durch die Poriferen des Untergrundes das Wasser zum Wallen gebracht werden. Diese starken Wasserwirbel haben scheinbar entgegengesetzte Wirkung. Sie erschweren durch diese dauernde Wasserbewegung und durch die Filtrierung des Wassers die Sedimentation auf der Unterlage, sie fördern diese aber gleichzeitig: 1. Durch die Bindung von Sandkörnern, Schlick und Organismenresten durch Schleimstoffe zu größeren Kotballen; 2. durch die Verwertung suspendierten Materials zum Gehäusebau (so können Sabellaria und Fabricia durch ihre Röhren enorme Massen Sand anhäufen); 3. durch die Bildung eines Lückensystems zwischen den Tieren, in deren Stillwassergebieten nun doch reichlich Material gelagert und festgehalten wird, das seinerseits von Cyanophyceen durchwachsen wird. So kann sich auf Felsboden ein "Steinschlick“ durch die Tätigkeit von Organismen bilden, der ohne diese nie entstanden wäre. In Brasilien fand ich in der Lagune von Cananea supralitoral eine bis $6 \mathrm{~mm}$ dicke Schicht, die aus Mud und kleinen Sandkörnern bestand, sie war ditht durchsetzt von Cyanophyceen. In ihr lebten Polychaeten (Lycoridae), Mückenlarven, Harpacticiden, Nematoden, auf ihr Schnecken (Littorina), Oncidiacea etc.

Auch nach dem Absterben der Tiere können die Gehäuse noch einen biologischen Einfluß haben. In leeren Balaniden-Gehäusen der Gezeitenregion bleibt oft während der Niedrigwasserperiode Wasser, so daß diese leeren Schalen ein Mikro-Aquarium sind. Am Strand Brasiliens (San Sebastian) sah ich, wie kleine Krabben (Brachyuren) sich während der Ebbe hier „im Wasser“ aufhielten. Vielleicht spielen diese Kleingewässer 
für die Meerestiere eine ähnliche (wenn auch geringere) Rolle wie die Kleingewässer in Bromelien, Dipsacus, Baumhöhlen (Phytotelmen) für die limnische Tierwelt.

Tiefgreifender ist die Beeinflussung des Substrates durch seine endolithischen Bewohner, da sie durch ihre Gänge im Festboden einet neuen Strukturteil für die Besiedlung schaffen. Auch hier ist die Wirkung endolithischer Felsbewohner während ihrer Lebenszeit und nach ihrem Absterben sehr verschieden. DerPolychaet Polydora ciliata, der durch seine mechanische Bohrtätigkeit (vgl. Hemper 1957, 1960) Kalksteine und Kalkschalen durchlöchert, baut über der Offnung eine kleine Röhre aus angekitteten Fremdkörpern. Diese Mudschicht aus Wurmröhren wird nach DEN HARTOG (1959, p. 53) $1-2 \mathrm{~cm}$ hoch und ermöglicht die für diese Region zuständige Algenassoziation (Polysiphonieto - Chaetomorphetum). Mehrere Arten meiden die mit Polydora besetzten Steine, zum Beispiel Phyllophora membranifolia, Chaetomorpha aerea und Abnfeltia pilcata; andere bevorzugen diese Polydora-Zone, zum Beispiel Acrosiphonia centralis, Petalonia fascia, Ulva lacuca. Sind aber von Polydora durchbohrte Steine nicht mehr von den Polychaeten selbst bewohnt, so entsteht durch sie ein neuer Strukturteil, der sehr wohl für Tiere der Mikrofauna einen bevorzugten Siedlungsraum abgeben kann. Untersuchungen hierüber liegen meines Wissens noch nicht vor. Sehr reich an Arten und Individuen ist das Sandröhrensystem abgestorbener Sabellariiden-Bauten. An der atlantischen Felsküste Brasiliens fand ich in diesen Röhren im Gezeitengebiet zahlreiche Polychaeten (Lycoridae, Syllidae, Sabellidae), Amphipoden, Isopoden, Mückenlarven, aber auch Elemente der Landfauna (Collembolen, Milben).

Viel deutlicher wird die Beziehung zwischen Struktur des Lebensraumes und Besiedelung in der $\mathrm{S}$ andregion. Seitdem ich vor 40 Jahren auf die merkwürdige reiche Fauna im Sand aufmerksam wurde, ist diese „interstitielle" Fauna des Sandbodens von vielen Seiten intensiv erforscht worden. In der Tat lebt hier eine bisher fast vollkommen übersehene Fauna, und die meisten Entdeckungen neuer Arten, Gattungen und Familien im Meeresraum hat die Untersuchung der Sandbiotope erbracht (vgl. Remane 1940, 1952, Delamare-Deboutteville 1960). Gegenüber den Hartböden und den Weichböden (Mud, Schlick) enthält der Sand einen neuen Strukturteil: das Lückensystem zwischen den Sandkörnern (Interstitium, Mesopsammal). In der Tat enthält dieses die reichste Fauna des Sandbodens (Abb. 1). Dieses Lückensystem ermöglicht eine vagile Mikrofauna nicht nur an der Oberfläche, sondern auch in der Tiefe des Sandbodens. Es ist offensichtlich, daß dieses Lückensystem in seinem Volumen durch die Korng röße des Sandes beeinflußt wird.

Schon bei der ersten Einteilung der Bodencoenosen der Kieler Bucht (REMANE 1933) wurde eine Halammobydra-Coenose für den groben Sand und eine Turbanellabyalina-Coenose für den feineren Sand aufgestellt. Besonders die Mikrofauna der Mittel- bis Grobsande (entsprechend dem Amphioxus-Grund) ist wesentlich reicher und audh durch zahlreiche Differentialarten von den Feinsandgebieten unterschieden. Das gilt für Copepoden, Ostracoden, Turbellarien, Gastrotrichen, Archianneliden (Polygordius, Protodrilus, Nerillidium), Nematoden, Ciliaten und andere (vgl. Ax 1951, 1954, Delamare-Deboutteville 1960, Dragesco 1960, Gerlach 1953, 1954, MeixNER 1938, Remane 1933). Natürlich sind solche Coenose-Bildungen ebenso wie die Pflanzenassoziationen nur Gruppenbildungen, deren Angehörige in ihren ökologischen Ansprüchen nicht voll identisch sind. Das gilt sogar für die Charakterarten und Dif- 
ferentialarten einer Assoziation. Jede Art wird voraussichtlich ihr spezifisches Optimum der Korngröße haben. Ausführlich hat dies Delamare-Deboutrevinte (1960) für Derocheilocaris remanei durchgeführt (Abb. 2). Gleichwohl existiert nach meinen Erfahrungen nicht ein kontinuierlicher, fließender Wechsel der Arten vom groben Kies bis zum

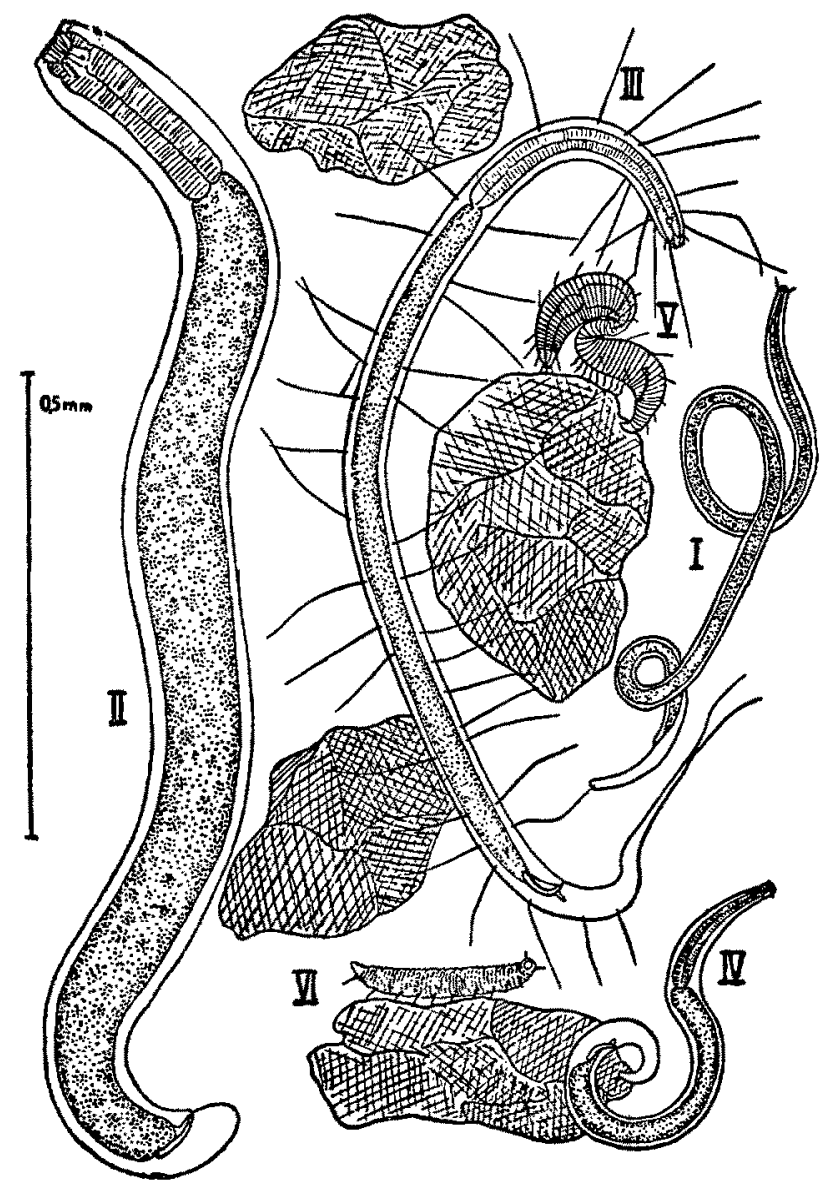

Abb. 1: Beispiele für die in den verschiedenen Lebensräumen des Brandungsstrandes vorkommenden Lebensformtypen unter den freilebenden Nematoden. I: Stemmschlängler (Axonolaimus tirrenicus); II: Bohrer (Halichoanolaimus obtwsicaudatus); III: Langbeborstete Form (Steineria mirabilis); IV: Schneller (Theristus beterospiculum); V: Spanner (Batbepsilonema pustulatum); VI Stelzer (Eudesmoscolex papillosus). Nach GerLach 1954

Feinsand und Schluff, sondern von Mittelsanden zu Feinsanden tritt ein schwellenartiger Artenwechsel ein. Die Fauna von Kies über Grobsande zu Mittelsanden erscheint ziemlich gleichartig, um beim Übergang zu Feinsanden rasch zu wechseln. Ich vermute die Ursache in der veränderten Zirkulation innerhalb des Lückensystems. In gröberem Material und daher breiterem Lückensystem ist die Wassererneuerung günstiger, die Tiefe des Lichteinfalls größer, falls es sich um Sande handelt. In feinerem Sande er- 
schweren die Kapillarkräfte die Zirkulation des Wassers in dem engen Kanalsystem, es sei denn im Gezeitengebiet, wo gerade die Feinsande bei Trockenlage und Insolation das Wasser der tieferen Schichten kapillar hochsaugen. Bei Zunahme der Materialgröße nimmt die interstitielle Fauna rasch ab, im Geröll aus kleinen Steinen bleiben als letzte eine Reihe Seriata, es kommen Formen der Makrofauna hinzu, wie Procerodes-Arten und in manchen Regionen auch Brachyuren und Isopoden. Eine Beziehung zwischen Korngröße und Besiedelung besteht nicht nur für die interstitielle Fauna, sondern auch

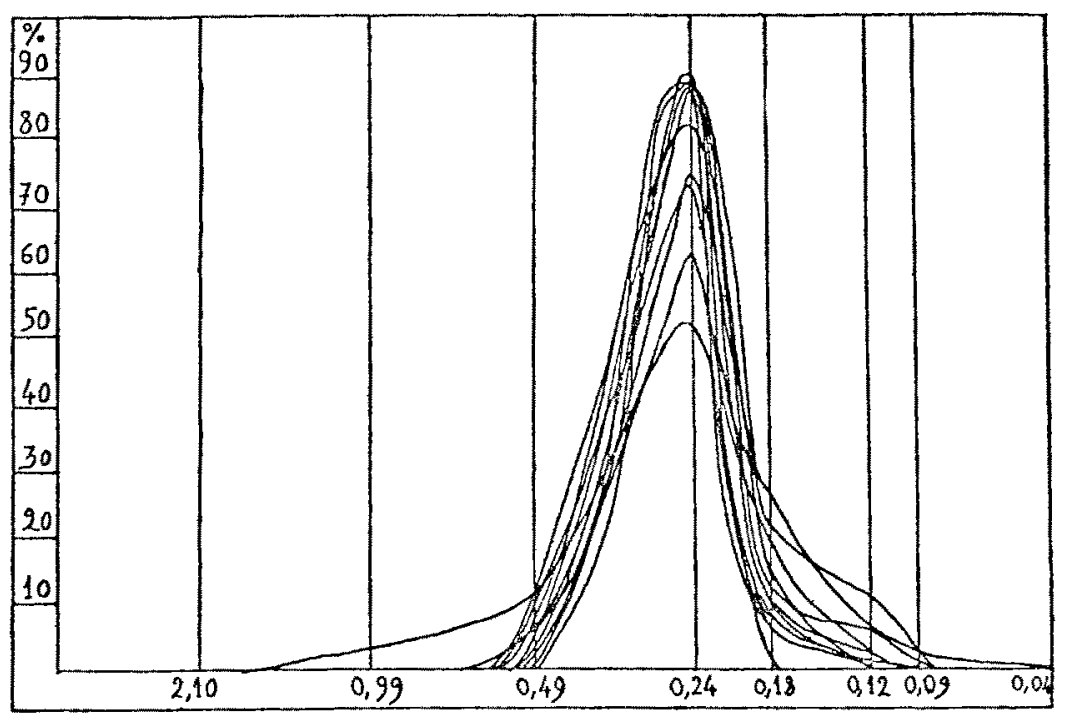

Abb. 2: Kornanalyse verschiedener Sande, in denen Derocheilocaris remanei gefunden wurde. (Nach Delamare 1953)

für die sich im Sand wühlend fortbewegenden oder sich in den Sand einwühlenden Arten, also für größere Nematoden, manche Acöla, Polychaeten (Opheliidae, Arenicola), manche Cumaceen (Pseudocuma, Lamprops), Amphipoden, Isopoden etc.

Streng genommen wirkt aber die Korngröße auf die Mikrofauna nur indirekt. Für die interstitielle Fauna ist das Lückensystem der Lebensraum. Seine Größe und Ausbildung hängt zwar von der Korngröße ab, wird aber entscheidend durch die Lage der Sandkörner (ob dicht oder locker) und durch ihre Form (rund, kantig, plattenartig wie etwa viele Schillteile) beeinflußt. Wie variabel aber die Packung der Sandkörner ist, zeigt uns jeder Meeresstrand. Neben Zonen, in denen der Fuß tief einsinkt, gibt es Regionen, die durchaus hart wirken. Beide Bereiche liegen am Brandungsstrand dicht nebeneinander. Am Prallhang ist der Sand dicht gepackt und arm an Tieren, am Fuße des Prallhanges in der Region der Mikroquellen und der Otoplanenzonen bei lockerer Packung des Sandes überaus reich. Da die Lagerung der Sandkörner von der Wasserbewegung abhängt, ist sie an gleicher Stelle wenigstens in den oberflächlichen Lagen nicht konstant; gleichzeitig aber auch nicht die Korngröße, da stärkerer Wellengang die kleineren Körner aufwirbelt und abtransportiert, die 
gröberen Materialien zurückläßt, ein Vorgang, der durchaus reversibel ist. Wir müßten also die Dimensionen und die Struktur des Lückensystems messen. Das ist aber bei unserer Art der Probenentnahme schwer durchführbar, da bei dieser, besonders wenn sie durch Stoßröhren erfolgt, doch das Lückensystem leicht gestört und verändert wird. Die Erschütterung des Sandgefüges während der Probenentnahme kann noch einen anderen unerwünschten Nebeneffekt haben, wenn man das Vorkommen der interstitiellen Fauna in verschiedenen Tiefenzonen unterhalb der Bodenoberfläche untersuchen will. Eine große Zahl von Arten reagiert auf Erschütterung durch geotaktische Fluchtbewegungen in die tieferen Schichten. Diese Reaktionen sind oft sehr intensiv und bei Turbellarien (Otoplaniden, Acölen), Copepoden, Gastrotrichen und anderen deutlich. Nach der Probenentnahme findet man diese Arten nicht in ihrem normalen Lebensbezirk, sondern in ihrem Fluchtraum oder in einem Bezirk ihrer Vertikalwanderung.

Für die Tiere, die sich in den Sand eingraben oder sich in ihm wühlend fortbewegen, spielt die Verschiebbarkeit des Substrates eine wichtige Rolle. Sie ist in Zonen mit reicher Wellenbewegung in den oberen Schichten meist groß, und hier finden wir das Maximum sandbewohnender vagiler Amphipoden, Isopoden, Cumaceen (Bathyporeia-Zone), in stilleren Gebieten dominieren die Röhrenbauer (Arenicola, Corophium und andere). Daß Arten dieser Makrofauna das ihnen zusagende Substrat aktiv aufsuchen, beziehungsweise inadäquate Böden bald wieder verlassen, hat WIESER (1956) in schönen Experimenten an Cumaceen gezeigt.

Ein weiterer Strukturkomplex ist die Schichtung des Substrates. Aus der Vegetationskunde ist bekannt, daß der Ortstein, jene mineralische Verkittung im Sandboden in einer gewissen Tiefe, den Pflanzenwuchs tiefgreifend beeinflußt. An Stelle des echten Waldes wächst hier nur die Buschform des Krattes, weil der Ortstein ein Eindringen der Baumwurzeln in die Tiefe verhindert. In gleicher Weise genügt am sandigen Meeresstrand eine nur wenig dicke Ton- oder Schlammschicht im Boden, um die Fauna der Küstengrundwasser von einer Besiedelung dieser Regionen auszuschlieBen (Delamare-Debouttevili.e 1960, Remane 1952). Oberhalb dieser Tonschicht ist eine interstitielle Fauna möglich, unter ihr nicht. Der Grund ist der entgegengesetzte wie beim Ortstein. Dieser hindert die Pflanzen, die Nahrungs- und Wasserbestände unterhalb des Ortsteins aufzuschließen, am Strande verhindert die Tonschicht die Versorgung der unter ihr liegenden Lückenräume mit frischem Wasser und Nahrung von oben. Lagert sich einer Sandfläche eine Schicht mineralischer Feinbestandteile (Ton, Kalk) auf, so hat dies für die interstitielle Fauna des Sandes katastrophale Folgen. Sie wirkt nicht nur durch die Abschirmung der Erneuerung des Porenwassers, sondern durch Verklebung der Haftorgane interstitieller Tiere. Da gerade diese besonders reich mit Haftorganen versehen sind, werden sie dadurch unfähig zur Fortbewegung und verkommen bald in diesem fremden Milieu. Im Verfolg meiner Untersuchungen über die interstitielle Fauna erwartete ich einen besonderen Reichtum in den Korallensanden. Meine Erwartung wurde enttäuscht, da sie meist mit einer Schicht feinen Kalkes bedeckt oder durchsetzt sind, die die Existenz einer Mikrofauna unmöglich machen. Besonders an Riffen mit Kalkalgen (Nulliporen) war dies auffällig. Erst als ich Proben von der Außenseite des Riffes nahm, wo die Wasserbewegung die Sedimentierung des Feinmaterials verhinderte, fand ich die erwartete reiche Fauna. Der Boden bestand hier 
aus Foraminiferenschalen, kleinen Trütnmern von Korallen und kleinen Molluskenschalen.

Die Wirkung einer Uberschichtung des Bodens durch "Vorsediment" ist übrigens ganz verschieden, je nachdem, ob die Schicht aus flockigem organischen Material oder aus mineralischen Bestandteilen besteht. Für die letztere gilt die eben geschilderte destruktive Wirkung auf die Mikrofauna des Bodens; der mit Bakterien beladene organische Detritus bietet eine günstige Nahrungsgrundlage auch für Tiere des $\mathrm{Me}$ sopsammons; ferner behält der Detritus eine flockige Struktur, enthält also selbst wie der Sand ein Lückensystem. In dieses wandern Tiere der interstitiellen Fauna hinein (Copepoden, Nerillidium, Gastrotrichen), so daß eine Uberschichtung mit flockigem Detritus eine positive Wirkung für viele der interstitiellen Bewohner haben kann. Diese Tatsache machte mir einen zunächst unverständlichen Befund verständlich. Bei meinen Untersuchungen in der Kieler Bucht und im Wattenmeer fand ich im Schlidk kaum bewimperte Organismen (Ciliaten, Turbellarien), während Arthropoden (Copepoden, Ostrakoden, Halacariden) reich vertreten waren. Bei ihren Untersuchungen an der Küste Grönlands fanden STEINBöcK und REISINGER sehr wohl Turbellarien in größerer Tiefe auf Schlammboden. Später fand ich auch in der Kieler Bucht (Stoller Grund-Rinne) Stellen mit einer reichen Fauna an bewimperten Tieren, aber an einer Stelle, an der sich organischer Detritus vermengt mit Algentrümmern auf der Schlammschicht angehäuft hatte. Der positive Effekt einer solchen Oberlagerung des Bodens mit einer Schicht aus Resten von Lebewesen hat aber seine Grenze. Nach der Frühjahrswucherung von Planktondiatomeen können sich auf dem Boden der Kieler Bucht in Senken große Massen von abgesunkenen Diatomeen zu einer dicken lockeren Lage anhäufen. Diese kann zur Vernichtung der Bodenfauna fübren. Dabei sind offenbar zwei Faktoren wirksam. Die Sauerstoffzehrung bei der Verwesung der Planktonmassen vernichtet zum größten Teil die Bodenfauna. In diesem Falle tritt eine Schwärzung der Bodenoberfläche, wie sie von $\mathrm{O}_{2}$-armen und $\mathrm{H}_{2} \mathrm{~S}$-reichen Gebieten bekannt ist, ein. Ferner bewirkt die Masse an stachligen Diatomeen (Chatoceros), daß die Strudler unter den Bodentieren nach wiederholten Versuchen sich einziehen und so die Nahrungsaufnahme bei gedecktem Tisch aufgeben. Selbst wenn dieses Vorsediment aus groben Resten abgestorbener Zostera-Blättern besteht, können sich Bodentiere in dieser lokalen Masse ansiedeln: Schnecken (Acera, Philine), Halicryptus, Amphipoden (Pontoporeia, Parambus, Microdeutopus), sogar Arten des Mesopsammon (das Gastrotrich Macrodasys buddenbrocki wurde hier zuerst gefunden). Dieses Vorsediment bleibt an manchen Stellen längere Zeit angehäuft liegen, an anderen ist es nur vorübergehend vorhanden und wird bald aufgezehrt oder aufgewirbelt und an andere Stellen transportiert. Da im Meer die Wasserbewegung am Grund große Bezirke umfaßt - wie Rippelmarken in $3000 \mathrm{~m}$ Tiefe zeigen -, gilt die wechselnde Lagerung des Präsediments für weite Teile des Meeresbodens, während im Süßwasser sein Ubergang in echtes Sediment viel ungestörter ist, wenigstens überall, wo ruhige Becken in Seen und Tümpeln vorhanden sind. Seine genauere Untersuchung ist noch empfehlenswert. Leider wird es bei Probenentnahmen mit Dredgen und Stoßröhren gestört.

Ubrigens wird eine andere Art von Präsediment von dauerhaten Kotpillen ( $\mathrm{Hy}$ drobia!), Fraßkugeln von Krebsen (Dotilla, Uca, Ocypoda) und von abgerollten Kugeln zerstörter Sabellarienbauten geliefert. Während die Geologen ihnen schon Auf- 
merksamkeit geschenkt haben, wissen wir über die biologische Auswirkung dieser Auflagerungen kaum etwas. Wir sind nun schon in die Frage hineingeraten, wie die Organismen des Bodens seine Struktur verändern. Die Umschichtung beziehungsweise Entschichtung des Bodens durch wühlende Organismen (Branchiostoma, Arenicola und andere) ist durch Geologen und Aktuopaläontologen eingehend untersucht worden (SCHÄFER 1962).

Auf der anderen Seite können Organismen eine bisweilen dauerhafte Haut auf der Bodenoberfläche bilden. Auch Sand- und Schlickböden können eine „Vegetationsdecke“ tragen. Sie besteht allerdings nicht in einem Aufwuchs (Phytal) aus Busch-oder Strauchalgen, sondern aus einzelligen Pflanzen, speziell Diatomeen, bodenlebenden

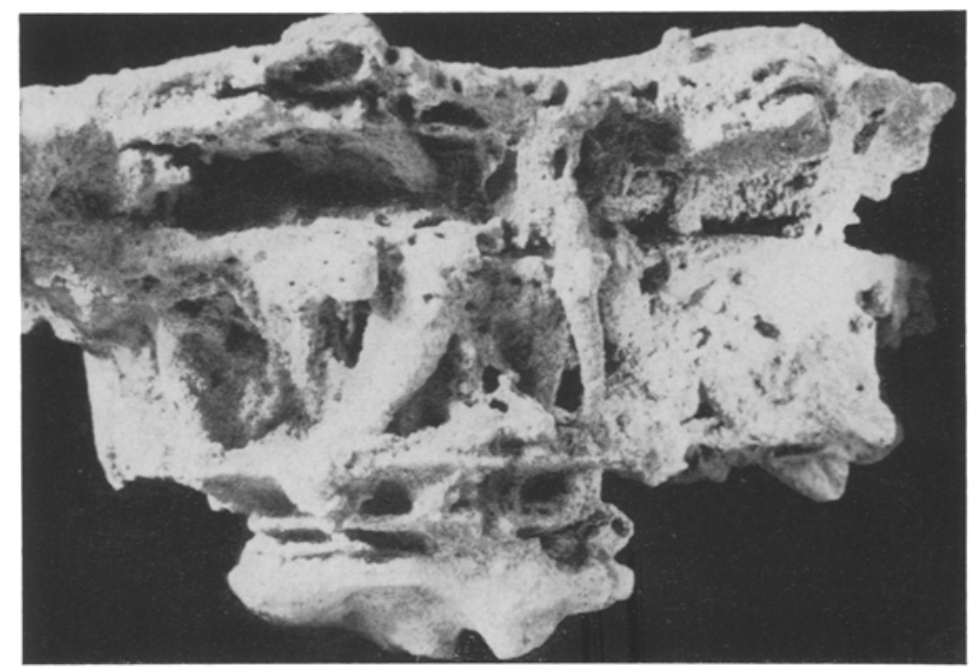

Abb. 3: Feinsandiger Substratbezirk, welcher durch die Tätigkeit von Würmern so verfestigt wurde, daß er der Zerstörung durch Wellen zu widerstehen vermag; der nicht durch Schleim gebundene Teil des Sedimentes ist ausgewaschen

Peridineen und im Gezeitengebiet auch Chlorophyceen. In gröberen Mittelsanden, die viel von Wasser bewegt und durchspült werden, sitzen sogar auf jedem Sandkorn mehrere Diatomeen, soweit es der Lichteinfall gestattet, sie bilden aber keine Vegetationsdecke. Auf feineren Sanden entstehen aber ganze Diatomeenflächen, die bekannten Diatomeensande. Hat ein Sturm die Sandschicht umgewirbelt und eine neue glatte Oberfläche geschaffen, so treten die Diatomeen- und Peridineenüberzüge zunächst in Flecken auf, wie Bakterienkulturen auf einem Nährboden. Sie breiten sich aus und vermehren sich, bis eine braune Decke entsteht, deren Gasblasenbildung die rege Assimilation verrät. In dieser Diatomeenschicht erfolgt eine Massensiedlung zahlreicher Arten der Mikrofauna des Sandes (Turbanella byalina, Neodasys, Batillipes, Acöla). Sie bildet aber noch keine dauerhafte Haut auf dem Sediment. Nach einem Sturm ist die Diatomeendecke verschwunden. An Stellen, an denen man am Tage vorher noch in $1000 \mathrm{~cm}^{3}$ Sand viele Hunderte von Gastrotrichen und Batillipes gefunden hat, findet man nach einem Sturm in einer gleichgroßen Probe vom gleichen Ort gar keine mehr, 
bis die Diatomeendecke regeneriert ist. Anders wird die Situation, wenn die Sandkörner der Oberfläche durch Schleimstoffe verkittet werden. Das kann durch kriechende Schnecken, durch das Sekret röhrenbauender Würmer, zum Teil auch durch Diatomeen, besonders aber wenn Cyanophyceen mit ihren Schleimröhren die Sandkörner umspinnen und verkitten, erreicht werden. Bei Anwesenheit einer reichen Lage von organogenem Detritus wird auch dieser zu einer Schicht verwebt. Solche Lagen können sehr resistent sein und wie ein Teppich vom Boden abgerollt werden (vgl. WoHLENBERG 1937, 1939).

Sie bieten einen wesentlichen Schutz gegen die Zerstörung des Bodens durch Wasserbewegung. Im Farbstreifenwatt, wo die austrocknende Wirkung von Sonne und Wind an der direkten Bodenoberfläche während der Trockenlage die Besiedlung an der Oberfläche selbst verhindert, bildet sich ein solcher Teppich einige Millimeter unter der Oberfläche (Schulz 1936). Einen sehr resistenten Teppich bilden auch die Massensiedlungen von Pygospio elegans, wenn die vertikalen, mit Schleim austapezierten Röhren der Tiere dicht beieinander liegen. Diese von Lebewesen geschaffene Decke auf Sand- oder Schlammboden kann in ariden Klimabezirken so fest werden, daß sie der Zerstörung durch die Wellen widersteht oder nur in größeren Stücken verfrachtet wird. In dem am Strand des Mittelmeeres bei St. Maries de la mer aufgelesenen Stück (Abb. 3) sind die Oberflächenschichten und die von Würmern verkitteten Röhrengänge in mehreren Etagen erhalten; unverkitteter Sand ist fortgespült. Die Verkittung ist derart, daß sessile Tiere wie Bryozoen und Muscheln dieses neue Substrat besiedeln.

Wie aus diesem Beispiel hervorgeht, können die Arten der Makrofauna einschließlich der Röhrenbauer eine über sie gelagerte Sedimentschicht durchwühlen und wieder ihre Normallage zur Oberfläche einnehmen. Viele Beispiele hierfür gibt SchärER (1962). Die Wirkung einer Überlagerung oder Zwischenschichtung durch ein anderes Sediment hat also für die Makrofauna nicht die destruktive Wirkung wie für einen Teil der Mikrofauna.

Uber die Wirkung der Schleimschicht auf die unter ihr lebende Fauna ist übrigens wenig bekannt. Daß in einer Diatomeenschicht eine Massenansammlung von Tieren der interstitiellen Fauna häufig vorkommt, wurde bereits erwähnt. In den Detritusreichen Gebieten des Farbstreifen-Wattes liegt unter der Grïnschicht aus Cyanophyceen eine Rotschicht aus Purpurbakterien, darunter eine Schwarzschicht ohne Sauerstoff. Eine der merkwürdigsten Schichtungen hat WoHLEnBerg $(1937,1939)$ im Gezeitengebiet der Nordsee gefunden. Hier dringt während des Niedrigwassers Luft in den Porenraum lockerer Sande, während der folgenden Überflutungen bleibt aber ein Teil der Luft in einer schwammigen Schicht im Sand und bildet den Porenlutthorizont. Er bildet den Lebensraum bestimmter Insekten, zum Beispiel des Käfers Diglotta mersa und der Dipterenlarve Lispa caesia. Über dieser lufthaltigen Etage können Meerestiere, etwa Spioniden, leben.

Eine sehr starke Beeinflussung des Bodens durch ein Lebewesen zeigen die von Seegräsern (Zostera, Posidonia, Diplanthera) besiedelten Flächen. Das Rhizomgeflecht mit seinen Wurzeln festigt den Boden. Der dichte Blattbestand wirkt für Sedimentablagerung fördernd. Dadurch wird die bewachsene Fläche buckelartig erhöht. Das läßrt sich im Gezeitengebiet sowohl an den Wuchsstellen von Zostera nana als auch an denen der Hydrocharitacee Diplanthera beobachten. Bei didhter Siedlung fängt die 
Seegraswiese stark Detritus, der hier $\mathrm{O}_{2}$-Mangel hervorrufen und eine Faulschlammschicht $z w i s c h e n$ den Blättern bilden kann, in der sich Bewohner Orarmen Muds, wie Halicryptus, Priapulus, Capitella und andere ansiedeln können. Dadurch wird am gleichen Ort durch das Seegras ein völliger Wechsel der Fauna und der Sedimentierung erreicht. Als durch eine Infektionskrankheit die Seegrasbestände vor etwa 30 bis 40 Jahren zerstört wurden, verschwanden mit dem Seegras die gesamte Fauna und der Mud. An seine Stelle traten wieder bewegte reine Sande. Darüber hinaus nahm der

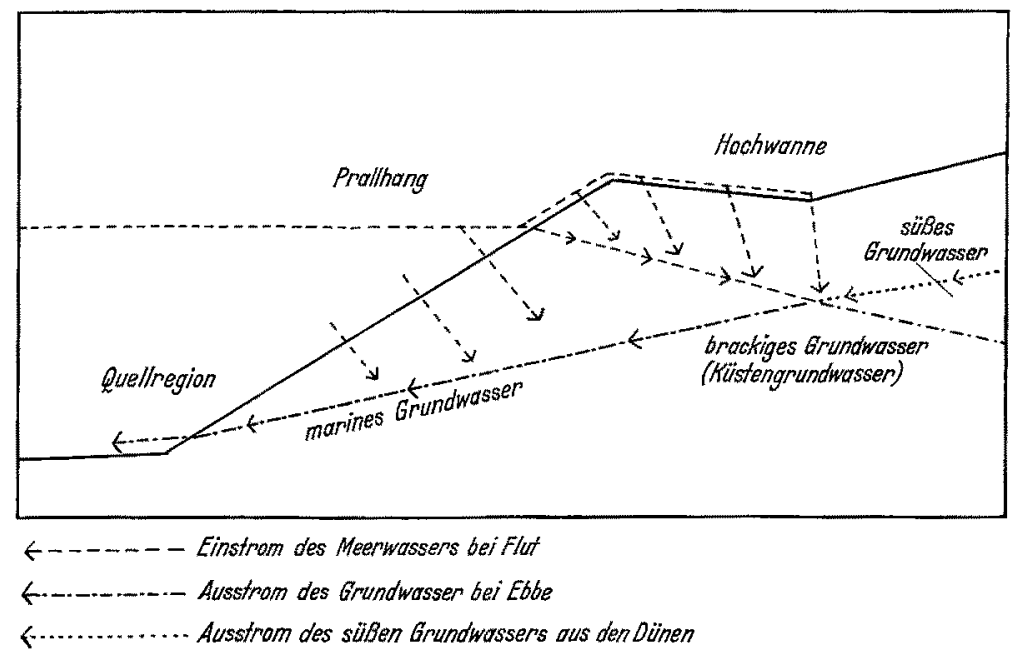

Abb, 4: Lebensbezirke im subterranen Litoralbereich der deutschen Küsten. (Nach GerLach 1953)

Bestand an kleinen Gastropoden (Zippora, Rissoa), Amphipoden (Gammarellus, Dexamine und andere) in der benachbarten Rotalgenzone stark ab. Leider konnte der $\mathrm{Ab}-$ lauf und die Ausdehnung dieser Faunenveränderung infolge des Krieges nicht näher verfolgt werden.

Die Beachtung der Struktur hat zur Klärung mancher Fragen geführt. Im Süßwasser fand man in den Höhlen eine recht eigenartige Fauna. Es begann mit der Entdeckung der Batbynella durch Vejpovsky (1882) in einem Brunnen auf dem Hradschin in Prag. Sie erschien als rezenter Abkömmling einer palaeozoischen Krebsgruppe und wurde vielfach bezweifelt, bis Chappurs $(1915,1927)$ sie in einem Brunnen bei Basel wiederentdeckte. Jetzt kennt man zahlreiche Arten der Gattungen Batbynella, Parabatbynella und Thermobatbynella in allen Erdteilen. Es folgten die Entdeckungen von kleinen neuen Crustaceen (Microcerberus, Microcharon, Bogidiella, Ingolfjella) durch KaRAMAN (1933), die Entdeckung des Archianneliden beziehungsweise Polychaeten Troglochaetus durch Delachaux (1921) etc. Die weitere Forschung zeigte, daß es sich hier nicht um Bewohner der Gewässer in Höhlen handelt, sondern um Arten des Spaltenwassers in wasserführenden Sanden und Kiesen, also um eine interstitielle Fauna subterraner Süßwässer. Die charakteristischen Arten dieser speziellen Fauna zerfielen in zwei Gruppen: a) alte Süßwasserbewohner, wie die Bathynellidae und wohl auch 
Parastenocaris, b) Arten, deren nächste Verwandte im Meer leben; limnische und marine Formen gehören dann meist der gleichen Gattung oder doch der gleichen Familie an (Microcerberus, Microcharon, Microparasellus, Bogidiella, Ingolfjella, Troglochaetus). Der Weg dieser marinen Emigranten in subterrane Süßwasser erschien zunächst rätselhaft. Nun erwiesen sich die marinen Vertreter als Bewohner des Lückensystems im Sande, gehörten also bereits im Meere der interstitiellen Fauna an. An sandigen Küsten dringt Meerwasser unter der Küste bis in "subterrane" Gebiete vor. Diese Küstengrundwasser (vgl. REMANE \& Schulz 1934) enthalten eine interstitielle Fauna, die neben marinen Formen auch spezielle Arten enthält. Eine Gesamtdarstellung dieser Fauna gab Delamare-Deboutreville (1960). Nun leben schon hier besonders solche Gruppen, die auch im subterranen Süßwasser erscheinen. Die Gleichheit der Struktur ermöglichte also vom Meeressand die Einwanderung in gleichstrukturierte limnische Bezirke, zum Teil wohl direkt über das Küstengrundwasser, das ja vom marinen Porenwasser über brackiges in süßes übergeht, zum Teil über die Ufersande von Flüssen (Abb. 4).

Von der Struktur aus wird auch die Verwandtschaft verständlich, die das Mesopsammon des Sandes mit der Fauna enger Pflanzenbestände (Moose, besonders Sphagnum, Fadenalgengeflecht des Supralitorals, Enteromorpha) zeigt. Auch diese haben und halten in ihren Interstitien Wasser, das ähnliche Existenzbedingungen gibt, wie das Porenwasser in Sanden. Ich erwähnte bereits, daß Arten des Sandlückensystems (Mesopsammon) in flockigen Detritus und sogar in angehäufte Reste toten Seegrases einwandern können, etwa Macrodasys buddenbrocki. Nun besiedeln die Tardigraden gerade diese Lückenräume im Sand, zwischen Fadenalgen und in Moosen. Nach der Zahl der Gattungen scheint der Meeressand ihr Ausgangsbiotop gewesen zu sein. Die Rädertiergattung Wierzejskiella findet sich in Sphagnum, interstitiell in Süßwassersanden und im Küstengrundwasser des Meeresstrandes. Die Copepodengattung Parastenocaris, die jetzt nach Noodt (mündl. Mitteilung) etwa 150 Arten umfaßt, fand man zuerst subterran im limnischen Bereich und in Sphagnum-Moosen, besonders in den Tropen. Man vermutete zuerst, sie habe im Tertiär oberirdisch bei uns gelebt und sei durch die Eiszeit in die unterirdischen Gewässer abgedrängt worden. Seitdem hat man sie auch in den Tropen zahlreich in Sanden als interstitielle Formen gefunden und auch in brackigen Gebieten des Küstengrundwassers am Meeresstrand ( $P$. vicesima, $P$. phyllura). Sie gehört also auch zur interstitiellen Fauna im weitesten Rahmen (vom Sand bis zu Pflanzeninterstitien) und belegt den leichten Biotopwechsel bei ähnlicher Struktur (NoodT 1955). Die Struktur des Meeresbodens ist also ein extrem wichtiger Faktor, der die Existenz und Ausbreitung ganzer Faunen bestimmt. Sie ist darïber hinaus ein Faktor, der neben der Nährstoffgrundlage die Biomasse des Bodens beeinflußt.

\section{ZUSAMMENFASSUNG}

1. Die Struktur des Meeresbodens kann Qualität und Quantität der Bodenfauna entscheidend beeinflussen. Sie ist ein wichtiger ökologischer Faktor, welcher die Existenz und Ausbreitung ganzer Faunen bestimmt. Die Bodenstruktur beeinflußt darüber hinaus neben der Nährstoffgrundlage die Biomasse des Bodens. 
2. In der Hartbodenregion kommt vor allem der Oberflächengestaltung (rauh oder glatt), der Dauerhaftigkeit der Substratoberfläche sowie ihrer chemischen Beschaffenheit entscheidende Bedeutung zu. Die Struktur kann zudem sekundär durch die Tätigkeit der Organismen modifiziert werden.

3. Besonders deutlich werden die Beziehungen zwischen der Struktur des Lebensraumes und dessen Besiedlung in der Sandregion. Im Lückensystem (Interstitium, Mesopsammal) des Sandbodens lebt eine reiche Fauna. Thre Existenz hängt weitgehend ab von der Größe und Ausbildung des Lückensystems. Diese wiederum ist eine Funktion der Korngröße und Kornpackung; sie ist der exakten Messung nur schwer zugänglich.

4. Von Wichtigkeit ist auch die Schichtung des Substrates. Ton- oder Schlammschichten zum Beispiel können ein Lückensystem unbewohnbar machen; dagegen kann eine Überschichtung mit flockigem Detritus eine positive Wirkung für viele Bewohner des Interstitiums haben.

5. Die Berücksichtigung der ökologischen Bedeutung der Bodenstruktur hat zur Klärung mancher tiergeographischer und systematischer Probleme geführt.

An dieser Stelle möchte ich Fräulein Dr. L. ScHürz für ihre freundliche Unterstützung bei der Beschaffung der Literatur danken.

\section{ZITIERTE LITERATUR}

Ax, P., 1951. Die Turbellarien des Eulitorals der Kieler Bucht. Zool. Jb. System. 80, 277-378.

- 1954. Die Turbellarienfauna des Küstengrundwassers am Finnischen Meerbusen. Acta zool. fenn. 81, 1-54.

Brandt, K., 1905. Uber die Produktion und die Produktionsbedingungen im Meere. Rapp. Cons. Explor. Mer 3, 1-12.

Chappuis, P., 1915. Batbynella natans und thre Stellung im System. Zool. Jb. System. 40, $147-176$.

- 1927. Die Tierwelt der unterirdischen Gewässer. In: Die Binnengewässer. Bd. 3. Schweizerbart, Stuttgart, 175 pp.

Delachaux, T., 1921. Un Polychète d'eau douce cavernicole. Bull. Soc. neuchâtel. Sci. nat. 45.

Delamare-Deboutteville, C., 1960. Biologie des eaux souterraines littorales et continentales. Vie et Milieu (Suppl. No) 9, 1-740.

DEN Hartog, C., 1959. The epilithic algal communities occurring along the coast of the Netherlands. Diss. Amsterdam.

Dragesco, J, 1960. Ciliés mésopsammiques littoraux. Trav. Sta. biol. Roscoff. 12, 1-356.

GerLACH, S., 1953. Die biozönotische Gliederung der Nematodenfauna an den deutschen Küsten. Z. Morph. Ökol. Tiere 41, 411-512.

- 1954. Die Nematoden des Sandstrandes und des Küstengrundwassers an der italienischen Küste. 2. OKkologischer Teil. Archo zool. ital. 39, 311-359. (Berichtig. d. Druckfehler in Bd. 40, 275-79 derselben Zeitschr.)

Hempes, C., 1957. Über den Röhrenbau und die Nahrungsaufnahme einiger Spioniden etc. Helgol. Wiss. Meeresunters. 6, 100-135.

- 1960. Ober das Festsetzen der Larven und die Bohrtätigkeit der Jugendstadien von Polydora ciliata. Helgol. Wiss. Meeresunters. 7, 80-92.

Karaman, S., 1933. Zwei neue Amphipoden, Balcanella und Jugocrangonyx. Zool. Anz. 103. Meixner, J., 1938. Turbellaria (Strudelwürmer). In: Die Tierwelt d. Nord- u. Ostsee. T. 4b. Akad. Verl. Ges., Leipzig. 
Noodt, W., 1955: Die Verbreitung des Genus Parastenocaris, ein Beispiel einer subterranen Crustaceen-Gruppe. Zool. Anz. (Suppl. Bd) 18, 429-435.

- 1957. Zur Okologie der Harpacticoidea des Eulitorals der deutschen Meeresküste und der angrenzenden Brackgewässer. Z. Morph. Ökol. Tiere 46, 149-242.

Precht, H., 1935. Epizoen der Kieler Bucht. Nova Acta Leopoldina N. F. 3, 405-477.

Remane, A,, 1933. Verteilung und Organisation der benthonischen Mikrofauna der Kieler Bucht. Wiss. Meeresunters. Abt. Kiel 21, 161-222.

- \& Schurz, E., 1934. Das Kïstengrundwasser als Lebensraum. Schr. Naturw. Ver. Scbl.Holst. 20, 399-408.

- 1940. Einführung in die zoologische Okologie der Nord- und Ostsee. In: Die Tierwelt d. Nord- u. Ostsee. T. 1a. Akad. Verl. Ges., Leipzig, 238 pp.

- 1952. Die Besiedlung des Sandbodens im Meere und die Bedeutung der Lebensformtypen für die Okologie. Zool. Anz. (Suppl. Bd) 16, 327-359.

SсHёFER, W., 1938. Bewuchs-Verteilung von Seepocken (Balaniden) im Gezeitengürtel. Natur Volk 68, 564-569.

- 1962. Aktuopaläontologie nach Studien in der Nordsee. (Senckenberg-Buch. 41.) Kramer, Frankfurt, 666 pp.

Schulz, E., 1936. Das Farbstreifensandwatt und seine Fauna. Kieler Meeresforsch. 1, 359-377.

SснӥTZ, L., 1961. Verbreitung und Verbreitungsmöglichkeiten der Bohrmuschel Teredo navalis L. und ihr Vordringen in den Nord-Ostsee-Kanal bei Kiel. Kieler Meeresforsch. 17, $228-236$.

SteINBöck, O., 1931. Nemertoderma bathycola nov. gen. spec. Vidensk. Medd. dansk. naturh. Foren. 90, 46-84.

VejDovsKX, F., 1882. Tierische Organismen der Brunnengewässer von Prag. Prag.

Wreser, W., 1956. Factors influencing the choice of substratum in Cumella vulgaris Hart. Limnol. \& Oceanogr. 1, 274-285.

Wohl.ENBerg, E., 1937. Die Wattenmeer-Lebensgemeinschaften im Königshafen von Sylt. Helgol. Wiss. Meeresunters. 1, 1-92.

- 1939. Die Nutzanwendung biologischer Erkenntnisse im Wattenmeer. Rapp. Cons. Explor. Mer 109 (Pt 3), 125-130.

\section{Diskussion im Anschluß an den Vortrag Remane}

JARKE: Zur Frage der Konservierung von Sedimentstrukturen wäre zu sagen: Es gibr heute im Kolbenlot und dem in Wilhelmshaven entwickelten Kastengreifer Geräte, mit denen sich abgesehen von gewissen Randverschmierungen - ungestörte Bodenproben entnehmen lassen. Die Bodenproben können mit bestimmten Gießharzen durchtränkt und gehärtet werden. Man kann dann von den künstlich "fossilisierten" Sedimentblöcken Anschliffe oder auch durchsichtige Schliffe herstellen und an Hand dieses Materials die Feinstrukturen der Sedimente untersuchen. Die Methode setzte bisher voraus, daß die Proben vor dem Tränken getrocknet wurden. Wie ich kürzlich erfuhr, soll jetzt in England ein Gießharz hergestellt werden, mit dem man Sedimente in naturfeuchtem Zustande - also unter Umständen in situ - härten kann.

WIESER: Zwischen Struktur und Chemismus des Bodens bestehen oft enge Beziehungen. So verändert die Anhäufung von lockerem Material in den Lückenräumen unter anderem die Durchlüftung und schaff sauerstoffarme Bereiche. Die Verbreitung von Bodentieren wird vermutlich auch durch derartige chemische Faktoren limitiert.

Remane: Ganz sicher spielt der Chemismus des Substrates eine Rolle. In diesem Zusammenhang möchte ich auf Beobachtungen eingehen, welche wir in Korallengebieten des Roten Meeres gemacht haben. Als wir einen Korallensand untersuchten, der fast ausschließlich aus Foraminiferen, Muschelschalentrümmern und aufgearbeiteten Materialien des dortigen Korallenriffs bestand, fanden wir eine typische interstitielle Fauna. Diese Fauna fehlte aber 
sofort, wenn in umfangreichem Maße Kalkalgen am Korallenriff beteiligt waren. Wir fanden dann viel feinere Ablagerungen des Korallensandes, die nahezu steril sind, weil die Lücken durch das Feinmaterial verstopft waren. Sauerstoffarme Zonen kann man besonders schön an der Nordseeküste auf jedem Sandwatt sehen. Ich erinnere an das Farbstreifen-Sandwatt auf Amrum, wo ja die Schwarzzone schon ein paar Millimeter unter der Oberfläche beginnt, und wo auch wieder durch Geflechtalgen Zirkulation und Chemismus verändert werden. 